\title{
Atherogenic remnant lipoproteins: role for proteoglycans in trapping, transferring, and internalizing
}

\author{
Robert W. Mahley1,2,3,4,5 and Yadong Huang1,2,3,6 \\ ${ }^{1}$ Gladstone Institute of Neurological Disease and ${ }^{2}$ Gladstone Institute of Cardiovascular Disease, San Francisco, California, USA. ${ }^{3}$ Department of Pathology, \\ ${ }^{4}$ Department of Medicine, ${ }^{5}$ Cardiovascular Research Institute, and ${ }^{6}$ Department of Neurology, UCSF, San Francisco, California, USA.
}

\begin{abstract}
Unraveling the mechanisms controlling remnant lipoprotein clearance is important, as these lipoproteins are highly atherogenic. The most critical molecule in this process is apoE, which mediates high-affinity binding of remnant lipoproteins to members of the LDL receptor (LDLR) family and cell-surface heparan sulfate proteoglycans (HSPGs), which have been shown to play major independent as well as cooperative roles in remnant lipoprotein clearance. While all the players may have been identified, our understanding of how they interact and function together continues to evolve. In this issue of the JCI, MacArthur et al. (see the related article beginning on page 153) demonstrated that HSPGs under normal physiological conditions are critically important in the clearance of remnant lipoproteins, independent of LDLR family members. The complexity of VLDL and chylomicron remnant clearance was exemplified by the studies of Jones et al., also in this issue (see the related article beginning on page 165). Despite defective clearance of LDL in mice with a deficiency in the adaptor protein controlling internalization of the LDLR, called autosomal recessive hypercholesterolemia (ARH), remnant lipoprotein clearance was not grossly abnormal. A likely explanation is that the abnormal LDLRs bind the remnants and then transfer them to another acceptor for internalization. While the studies clearly demonstrate that the LDLR-related protein 1 is not involved and suggest a role for an additional unidentified receptor, it remains a possibility that HSPGs are responsible for remnant uptake by hepatocytes in the presence of defective LDLR internalization.
\end{abstract}

\section{Remnant lipoproteins from the liver and intestine}

Remnant lipoproteins are cholesterol-rich particles that are generated during circulation by lipolytic processing of liver-synthesized VLDL and intestine-synthesized chylomicrons $(1,2)$. Remnants are extremely atherogenic lipoproteins $(3,4)$. Lipoprotein lipase (LPL) on endothelial cell surfaces in capillaries and, to a lesser extent, hepatic lipase (HL) in the liver hydrolyze the triglycerides of these particles and generate remnants that under normal

Nonstandard abbreviations used: ARH, autosomal recessive hypercholesterolemia; FH, familial hypercholesterolemia; GlcNAc, N-acetyl glucosamine; HL, hepatic lipase; HLP, hyperlipoproteinemia; HSPG, heparan sulfate proteoglycan; LDLR, LDL receptor; LPL, lipoprotein lipase; LRP, LDLR-related protein; Ndst1, GlcNAc $N$-deacetylase/ $N$-sulfotransferase 1.

Conflict of interest: The authors have declared that no conflict of interest exists.

Citation for this article: J. Clin. Invest. 117:94-98 (2007). doi:10.1172/JCI30889. circumstances are rapidly and efficiently cleared from the blood by hepatocytes, where they are catabolized. Lipoproteins (density < 1.006) induced by cholesterol feeding in animals or occurring in patients with type III hyperlipoproteinemia (HLP, a genetic disorder characterized by remnant lipoprotein accumulation in the blood), called $\beta$-VLDL, are a mixture of both VLDL and chylomicron remnants, and their accumulation results in impairment of remnant clearance $(1,2,4)$. The major components of remnants, including $\beta$-VLDL, are apoB100, apoB48, apoE, and the apoCs. ApoE is the critical ligand responsible for remnant lipoprotein clearance $(1,5)$. LPL and HL may also serve as ligands under some circumstances (6-9).

\section{Role of apoE in remnant binding and uptake}

Some of the first evidence defining a critical role for apoE in remnant clearance involved i.v. infusion of apoE in cholesterol-fed rabbits and the demonstration of accelerated clearance of $\beta$-VLDL remnants from the blood and their uptake by the liver (10). In transgenic mice and rabbits, overexpression of apoE accelerated remnant clearance (11-13). Furthermore, apoE was localized to the sinusoids in the space of Disse, and after infusion of remnant lipoproteins, apoE disappeared from the sinusoids and appeared in hepatocytes (14). ApoE-knockout mice have markedly defective remnant lipoprotein clearance $(15,16)$. Clearly, LPL and HL do not substitute for apoE in remnant clearance.

Compelling proof of apoE's role as a critical ligand for remnant lipoproteins came from understanding type III $\operatorname{HLP}(2,4)$. Type III HLP is associated with genetic mutations in apoE. The most common apoE variant [apoE2(R158C)] acts as a susceptibility factor that requires a second hit (obesity, hypothyroidism, menopause, etc.) to induce remnant lipoprotein accumulation $(2,4)$. The majority of apoE2 homozygotes actually have normal or even low plasma lipid levels and no significant impairment of remnant clearance. This apoE variant is also defective in binding to the LDL receptor (LDLR), the LDLR-related protein (LRP), and heparan sulfate proteoglycans (HSPGs). A transgenic animal model expressing this apoE variant can be induced to develop type III HLP (17).

A variant in which arginine at residue 142 is replaced by a cysteine [apoE(R142C)] has a dominant mode of type III HLP transmission with marked remnant lipoprotein accumulation and premature atherosclerosis (a second hit is not required) (18). Expression of this variant in transgenic mice causes remnant accumulation (19). This variant is defective in binding to LDLR, LRP, and HSPGs $(2,20)$.

However, critical information learned from studies of these apoE variants not only supports the importance of apoE 


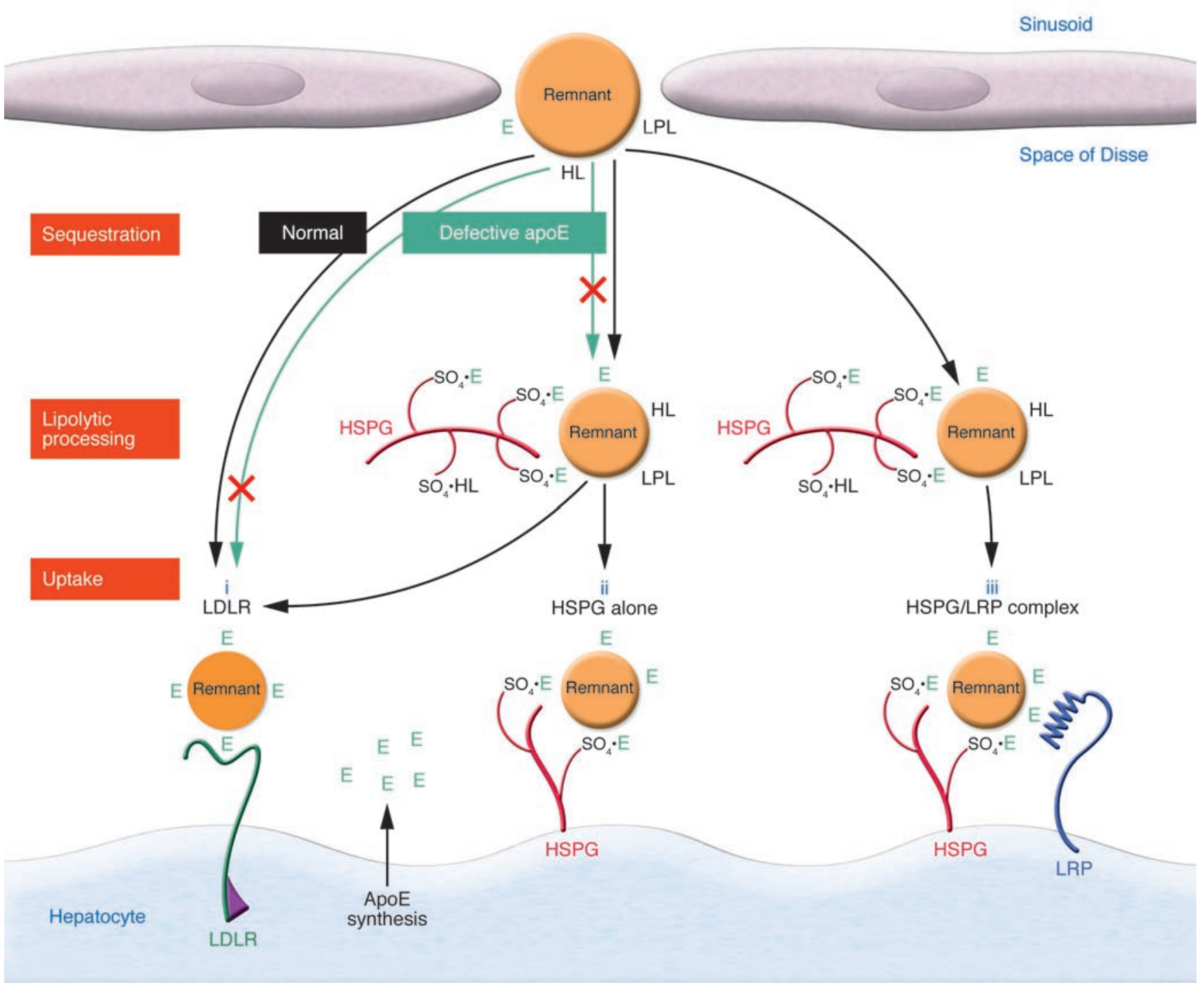

Figure 1

Remnant lipoprotein uptake is mediated by LDLR, HSPGs, and LRP. HSPGs are abundant in the matrix of the space of Disse and on the surface of hepatocytes. Sulfate groups $\left(\mathrm{SO}_{4}\right)$ on HSPGs interact with apoE, $\mathrm{LPL}$, and $\mathrm{HL}$. ApoE and possibly LPL and HL serve as ligands mediating sequestration, binding, and uptake of the remnants. Defective apoE, which occurs in type III HLP, displays variable defective binding to the LDLR and HSPGs compared with normal apoE. The LDLR, HSPGs, and the HSPG/LRP complex serve as receptors or coreceptors mediating remnant lipoprotein uptake (i-iii). E, apoE. Modified from the Journal of Lipid Research (1).

in remnant clearance but also provides insights into the critical receptors involved in remnant clearance. ApoE2 is markedly defective in binding to LDLRs $(<1 \%$ or $2 \%$ of normal apoE3 activity). However, apoE2 is characterized by a substantial capacity to bind to HSPGs (50\%-70\% of apoE3 activity) (20). This was one of the observations suggesting that HSPGs were involved, directly as a receptor or indirectly in complex with LRP, in the binding and uptake of remnants $(20,21)$. Remnants accumulate and type III HLP develops in apoE2 homozygotes only when the HSPG pathway is overwhelmed by processes that increase remnant production or decrease clearance, secondary to a second hit $(2,4)$.

Interestingly, the importance of the HSPG pathway in remnant clearance is also supported by data reported in studies of the role of the dominant variants of apoE that cause type III HLP, such as apoE(R142C), apoE(R145C), apoE(K146Q), and apoE Leiden (no second hit required) $(2,4,20)$. The dominant variants are markedly defective in HSPG binding ( $<5 \%$ of apoE3 activity), but their LDLR-binding activity is $25 \%-45 \%$ that of normal apoE3 (20). Defective binding of apoE variants to HSPGs in the dominant forms of type III HLP is the only factor consistently associated with remnant lipoprotein accumulation in these patients or in model systems. Even though these variants are able to bind LDLR with avidity, the impaired HSPG binding results in impaired remnant clearance and hyperlipidemia. Clearly HSPGs play a critical independent role in remnant clearance.

\section{Multiple steps involved in remnant uptake by hepatocytes}

We have envisioned that HSPGs in the space of Disse bring all the participants in remnant clearance together (Figure 1). The first step 


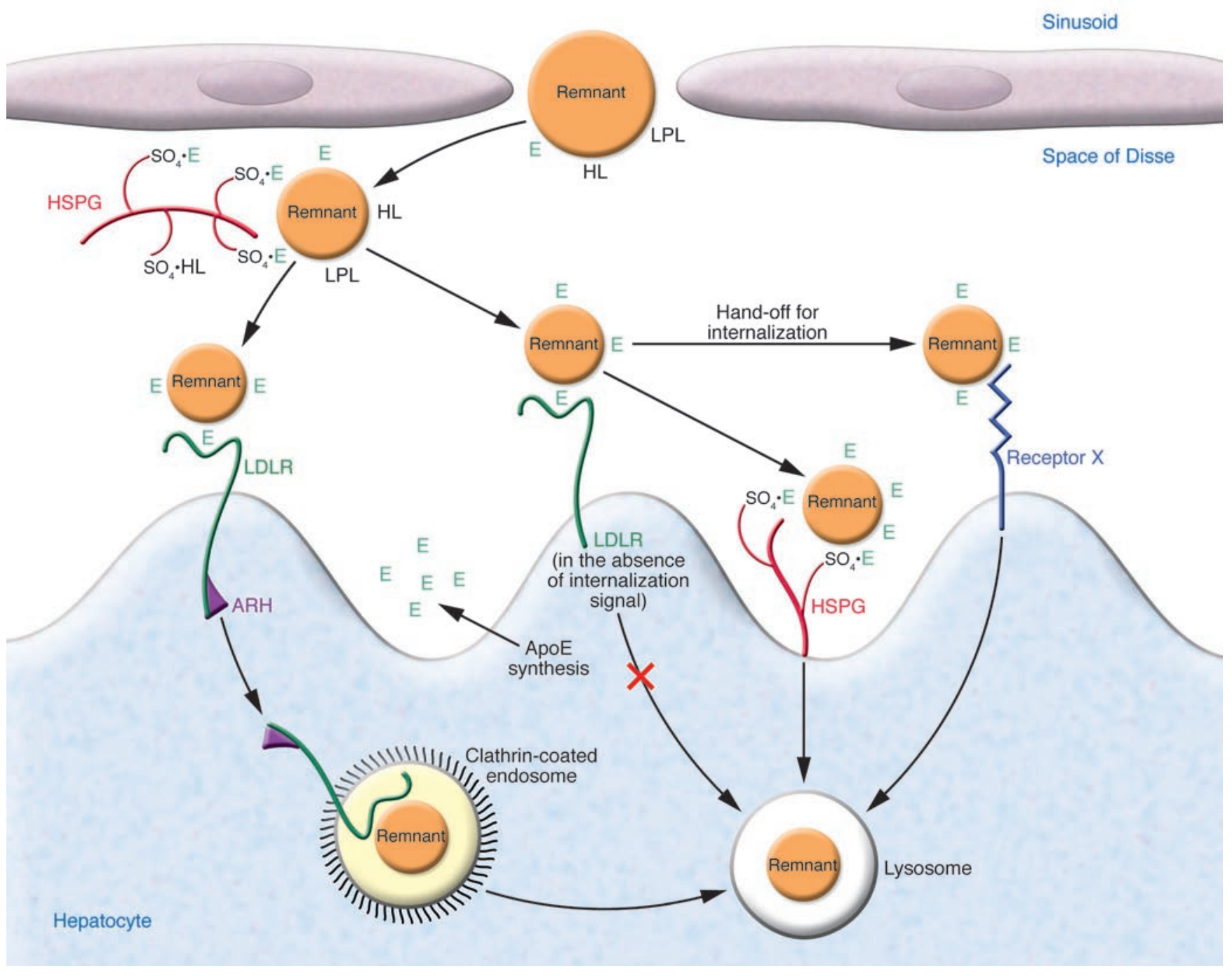

Figure 2

Under normal conditions in which the ARH is functional, the LDLR-dependent internalization of remnants by hepatocytes occurs. However, when the ARH is defective or absent, the remnants may bind to the LDLR but then are transferred to other cell-surface molecules for internalization. The acceptor may be HSPG or an additional unknown receptor (represented here as receptor X).

is sequestration. The remnants are captured by interaction with HSPGs, the binding being mediated primarily through apoE $(1,4,5)$. LPL and HL may continue their lipolytic processing, preparing the particles for uptake by hepatocytes and assisting in binding the particles to HSPGs $(1,7)$. ApoE synthesized and secreted by the liver serves as a reservoir for mediating binding and uptake. ApoE generated locally in the liver functions better than peripherally produced apoE in mediating remnant clearance (22). Internalization of the remnants involves several players.

\section{Remnant lipoprotein uptake by LDLR, LRP, and HSPGs}

For many years it has been known that the absence of normal LDLR activity leads to accumulation of LDL, but not of remnants $(23,24)$. While it has been clearly established that LDLR is involved in remnant clearance under normal physiological circumstances, there is significant LDLR-independent clearance, accounting for as much as $25 \%-50 \%$ of the hepatic remnant uptake (14) and suggesting that an additional mechanism (a remnant receptor) exists in the liver.

Herz and associates $(25,26)$ identified an additional member of the LDLR family, LRP1, as a major remnant receptor candidate. Whereas inactivation of LDLR in mice results in an increase in LDL and remnant levels, inactivation of LRP1 does not affect lipoprotein clearance, suggesting that in the presence of LDLR, LRP1 has little or no physiological function in remnant clearance. When both receptors are inactivated, there is a further accumulation of remnants, suggesting that LRP1 may serve as a backup system in the absence of LDLRs.

HSPG is the other major participant responsible for remnant clearance $(1,7,8$, $20,21,27-30)$. The critical role of HSPGs in lipoprotein uptake was established by our group $(1,21,27)$ as well as by Williams and Fuki (7). The importance of HSPGs in remnant binding and uptake was demonstrated by treating a variety of cells with heparinase. Heparinase treatment decreased remnant binding and uptake by $80 \%-90 \%$ in fibroblasts, familial hypercholesterolemia (FH) fibroblasts, and HepG2 
hepatocytes (21). The critical in vivo role for HSPGs was shown by i.v. infusion of heparinase into the portal vein of mice to release $\left[{ }^{35} \mathrm{~S}\right] \mathrm{HSPG}$ (which reduced the amount of HSPGs by $80 \%$ ). The clearance of apoE-enriched remnant lipoproteins was markedly reduced (28).

Previously, we suggested that HSPGs may form a complex with LRP to initiate remnant internalization by hepatocytes $(1,21,27)$. More recently, Wilsie and Orlando (31) coimmunoprecipitated the LRP and HSPG complex. Interestingly, their data suggest that LRP modulates the availability of VLDL-binding sites on HSPGs and that less binding and uptake of VLDL occur when the complex exists. This requires further study.

In this issue of the JCI, MacArthur et al. (32) have convincingly established that HSPGs serve not only as the LDLR-independent mechanism for remnant uptake, but may also play a role in LDL clearance. By inactivating GlcNAc N-deacetylase/ $N$-sulfotransferase $1(N d s t 1)$ in hepatocytes using the Cre-loxP system, these authors reduced sulfation of liver HSPGs by approximately $50 \%$. This resulted in impaired clearance of both VLDL and chylomicron remnants in the presence of LDLR and suggested that HSPGs represent the major LDLR-independent remnant receptor in the liver.

Interestingly and importantly, in contrast to LDLR knockout alone, the deletion of both NDST1 and LDLR resulted in an even more significant elevation of the levels of VLDL and intestinally derived (vitamin A-labeled) remnants and a marked delay in remnant clearance $(<25 \%$ cleared in 12 hours). These data demonstrate that LDLR and HSPGs are both involved in remnant clearance. Furthermore, the deletion of both LDLR and NDST1 resulted in higher LDL levels than the deletion of LDLR alone. This shows that HSPG participates in LDL clearance in the absence or possibly during downregulation of LDLRs. As illustrated in Figure 1, HSPGs appear to play a critical role in both LDL and remnant clearance, possibly sequestering (trapping) both classes of lipoproteins and then participating in handing off (transferring) the lipoproteins to LDLR or acting as receptors directly internalizing the lipoproteins. It has been clearly demonstrated that HSPGs can act as receptors for various ligands in other systems $(33,34)$.

An additional level of complexity in remnant clearance comes from the stud- ies by Jones et al. (35), also in this issue of the JCI. These authors suggest that the LDLR itself, under conditions where internalization is impaired, can transfer remnants to an additional receptor for uptake by the liver. Although Jones et al. clearly demonstrate that the LRP is not involved and suggest that HSPGs may not be involved, we would suggest that HSPGs have not been fully ruled out as the additional receptor responsible for remnant uptake.

The studies by Jones, Hobbs, and colleagues (35) build beautifully on the observations by Hobbs, Cohen, and their associates (36-38) describing an adaptor protein associated with LDLR that is critically important for LDL internalization by cells, including hepatocytes. Mutations in the adaptor protein are responsible for autosomal recessive hypercholesterolemia (ARH) (36), a form of hypercholesterolemia that is less severe than classical FH and is more responsive to lipid-lowering drugs. Despite the fact that LDL clearance rates are similar in $\mathrm{FH}$ and $\mathrm{ARH}$, plasma cholesterol levels are much lower in subjects with ARH.

Jones et al. (35) undertook to define the metabolic basis for this difference using transgenic mice. Two possibilities for the lower LDL levels with ARH mutations were considered: (a) increased VLDL remnant clearance, resulting in reduction of the formation of the end product (LDL) of VLDL metabolism; or (b) decreased hepatic production of VLDL. The first postulate proved to be correct.

Transgenic mice in which the ARH protein was inactivated ( $\mathrm{Ar} \mathrm{r}^{-/-}$mice) had LDL clearance rates similar to those of $\mathrm{Ldlr}^{-/-}$mice, despite having lower cholesterol levels (35). On a high-sucrose diet that induces VLDL production, the cholesterol levels in the $\mathrm{Arb}^{-/-}$mice were much lower than in the $\mathrm{Ldlr}^{-/-}$mice (301 versus $1148 \mathrm{mg} / \mathrm{dl}$ ); however, the triglyceride levels were 2 -fold higher in the $\mathrm{Ldlr}^{-/-}$mice than in the Arb-/- or WT mice. Whereas VLDL production rates were similar in the $\mathrm{Arb}^{-/-}$and $\mathrm{Ldlr}^{-/-}$mice, the clearance of $\left.{ }^{125} \mathrm{I}\right] \mathrm{VLDL}$ remnants was prolonged in $L d l r^{-/-}$mice (approximately 5-fold) compared with that in $\mathrm{Arb}^{-/-}$mice. These data support the concept that the ARH adaptor protein, while essential for LDL uptake by LDLR, might not be required for VLDL and remnant uptake.

However, when Jones et al. (35) attempted to define the ARH protein-associated mechanism responsible for the differential handling of LDL, VLDL, and remnants by LDLR, they encountered the complexity of remnant lipoprotein metabolism in the liver. While $\mathrm{Arb}^{-/}$cells expressed LDLRs on the surface of hepatocytes, they could not internalize LDL. However, remnants appeared to bind to LDLRs on $\mathrm{Arb}^{-/-}$cells, and then the remnants were taken up by the cells by a mechanism presumably independent of LDLR internalization, i.e., the bound remnants appeared to be transferred to another receptor for internalization (Figure 2). After ruling out several possible explanations for this result, Jones et al. (35) asked whether the remnants might be handed off to LRP for internalization. Deletion of LRP in $\mathrm{Arb}^{-/-}$mice did not alter the remnant clearance. Thus, the internalization of the remnants by $A r b^{-/-}$hepatocytes does not require LRP.

MacArthur et al. suggest (32) that removal of cell-surface HSPGs by heparinase treatment of primary $\mathrm{Arb}^{-/-}$ hepatocytes did not affect remnant internalization. To draw a firm conclusion, however, regarding the role of HSPGs in remnant uptake may require additional studies, including the in vivo infusion of heparinase through the portal vein into the liver of the transgenic mice or crossing $\mathrm{Arb}^{-/-}$mice with $\mathrm{NdstIf/f-Cre}{ }^{+}$mice to ascertain a role for HSPGs. However, if in fact this observation is confirmed, then there still may be an unidentified receptor to which the remnant particles are transferred for internalization (Figure 2).

\section{Conclusion}

The complexity of remnant lipoprotein metabolism results from several factors. As mammals evolved and consumed diets high in fat, physiological systems to handle the remnants that were generated also evolved. For example, apoE occurs only in higher-order mammals, undoubtedly at least in part to function in the clearance of remnants. Remnants are the largest of the lipoprotein particles (typically 400-800 ̊ in diameter), and they must enter the space of Disse, which is anything but a simple space. It is filled with HSPGs and the microvillus projections from hepatocytes extending into this matrix. Sequestration and capture of these large particles mediated by HSPGs is an essential first step that undoubtedly is occurring while lipolytic processing proceeds. Then these particles must reach the hepatocyte cell surface to bind to the receptors 
that will internalize them. It is reasonable to envision that the large HSPGs free in the matrix of the space of Disse and the HSPGs extending away from the cell surface of hepatocytes play a prominent role in passing off the large remnant particles to LDLRs or other receptors. Likewise, HSPGs serve to internalize the remnants directly. Future studies will continue to bring clarity to this process.

\section{Acknowledgments}

We thank Karl Weisgraber for critical reading of the manuscript, Sylvia Richmond for manuscript preparation, and John C.W. Carroll for graphics.

Address correspondence to: Robert W. Mahley, Gladstone Institute of Neurological Disease, 1650 Owens Street, San Francisco, California 94158, USA. Phone: (415) 734-2000; Fax: (415) 355-0820; E-mail: rmahley@gladstone.ucsf.edu.

1. Mahley, R.W., and Ji, Z.-S. 1999. Remnant lipoprotein metabolism: key pathways involving cell-surface heparan sulfate proteoglycans and apolipoprotein E. J. Lipid Res. 40:1-16.

2. Mahley, R.W., Huang, Y., and Rall, S.C., Jr. 1999. Pathogenesis of type III hyperlipoproteinemia (dysbetalipoproteinemia): questions, quandaries, and paradoxes. J. Lipid Res. 40:1933-1949.

3. Mahley, R.W., Weisgraber, K.H., Innerarity, T.L., and Rall, S.C., Jr. 1991. Genetic defects in lipoprotein metabolism. Elevation of atherogenic lipoproteins caused by impaired catabolism. JAMA. 265:78-83

4. Mahley, R.W., and Rall, S.C., Jr. 1995. Type III hyperlipoproteinemia (dysbetalipoproteinemia) the role of apolipoprotein $\mathrm{E}$ in normal and abnormal lipoprotein metabolism. In The metabolic and molecular bases of inherited disease. 7th edition. Volume 2. C.R. Scriver, A.L. Beaudet, W.S. Sly, and D. Valle, editors. McGraw-Hill. New York, New York, USA. 1953-1980.

5. Mahley, R.W. 1988. Apolipoprotein E: cholesterol transport protein with expanding role in cell biology. Science. 240:622-630.

6. Williams, K.J., et al. 1992. Mechanisms by which lipoprotein lipase alters cellular metabolism of lipoprotein(a), low density lipoprotein, and nascent lipoproteins. Roles for low density lipoprotein receptors and heparan sulfate proteoglycans. J. Biol. Chem. 267:13284-13292.

7. Williams, K.J., and Fuki, I.V. 1997. Cell-surface heparan sulfate proteoglycans: dynamic molecules mediating ligand catabolism. Curr. Opin. Lipidol. 8:253-262.

8. Ji, Z.-S., Dichek, H.L., Miranda, R.D., and Mahley, R.W. 1997. Heparan sulfate proteoglycans partici- pate in hepatic lipase- and apolipoprotein E-mediated binding and uptake of plasma lipoproteins, including high density lipoproteins. J. Biol. Chem. 272:31285-31292.

9. Zheng, C., Murdoch, S.J., Brunzell, J.D., and Sacks, F.M. 2006. Lipoprotein lipase bound to apolipoprotein B lipoproteins accelerates clearance of postprandial lipoproteins in humans. Arterioscler. Thromb. Vasc. Biol. 26:891-896.

10. Mahley, R.W., et al. 1989. Intravenous infusion of apolipoprotein E accelerates clearance of plasma lipoproteins in rabbits. J. Clin. Invest. 83:2125-2130.

11. Shimano, H., et al. 1994. Secretion-recapture process of apolipoprotein $\mathrm{E}$ in hepatic uptake of chylomicron remnants in transgenic mice. J. Clin. Invest. 93:2215-2223

12. Fan, J., et al. 1998. Increased expression of apolipoprotein $\mathrm{E}$ in transgenic rabbits results in reduced levels of very low density lipoproteins and an accumulation of low density lipoproteins in plasma. J. Clin. Invest. 101:2151-2164.

13. Huang, Y., et al. 1999. Overexpression of apolipoprotein E3 in transgenic rabbits causes combined hyperlipidemia by stimulating hepatic VLDL production and impairing VLDL lipolysis. Arterioscler. Thromb. Vasc. Biol. 19:2952-2959.

14. Ishibashi, S., et al. 1996. Role of the low density lipoprotein (LDL) receptor pathway in the metabolism of chylomicron remnants. A quan titative study in knockout mice lacking the LDL receptor, apolipoprotein E, or both. J. Biol. Chem. 271:22422-22427.

15. Plump, A.S., et al. 1992. Severe hypercholesterolemia and atherosclerosis in apolipoprotein E-deficient mice created by homologous recombination in ES cells. Cell. 71:343-353.

16. Zhang, S.H., Reddick, R.L., Piedrahita, J.A., and Maeda, N. 1992. Spontaneous hypercholesterolemia and arterial lesions in mice lacking apolipoprotein E. Science. 258:468-471

17. Huang, Y., Rall, S.C., Jr., and Mahley, R.W. 1997. Genetic factors precipitating type III hyperlipoproteinemia in hypolipidemic transgenic mice expressing human apolipoprotein E2. Arterioscler. Thromb. Vasc. Biol. 17:2817-2824.

18. Horie, Y., Fazio, S., Westerlund, J.R., Weisgraber, K.H., and Rall, S.C., Jr. 1992. The functional characteristics of a human apolipoprotein $\mathrm{E}$ variant (cysteine at residue 142) may explain its association with dominant expression of type III hyperlipoproteinemia. J. Biol. Chem. 267:1962-1968.

19. Fazio, S., Lee, Y.-L., Ji, Z.-S., and Rall, S.C., Jr. 1993. Type III hyperlipoproteinemic phenotype in transgenic mice expressing dysfunctional apolipoprotein E. J. Clin. Invest. 92:1497-1503.

20. Ji, Z.-S., Fazio, S., and Mahley, R.W. 1994. Variable heparan sulfate proteoglycan binding of apolipoprotein $\mathrm{E}$ variants may modulate the expression of type III hyperlipoproteinemia. J. Biol. Chem. 269:13421-13428.

21. Ji, Z.-S., et al. 1993. Role of heparan sulfate proteoglycans in the binding and uptake of apolipoprotein E-enriched remnant lipoproteins by cultured cells. J. Biol. Chem. 268:10160-10167.

22. Raffaï, R.L., et al. 2003. Hepatocyte-derived apoE is more effective than non-hepatocyte-derived apoE in remnant lipoprotein clearance. J. Biol. Chem.
278:11670-11675.

23. Kita, T., et al. 1982. Hepatic uptake of chylomicron remnants in WHHL rabbits: a mechanism genetically distinct from the low density lipoprotein receptor. Proc. Natl. Acad. Sci. U. S. A. 79:3623-3627.

24. Rubinsztein, D.C., et al. 1990. Chylomicron remnant clearance from the plasma is normal in familial hypercholesterolemic homozygotes with defined receptor defects. J. Clin. Invest. 86:1306-1312.

25. Herz, J., and Willnow, T.E. 1995. Lipoprotein and receptor interactions in vivo. Curr. Opin. Lipidol. 6:97-103.

26. Rohlmann, A., Gotthardt, M., Hammer, R.E., and Herz, J. 1998. Inducible inactivation of hepatic LRP gene by Cre-mediated recombination confirms role of LRP in clearance of chylomicron remnants. J. Clin. Invest. 101:689-695.

27. Ji, Z.-S., Fazio, S., Lee, Y.-L., and Mahley, R.W. 1994. Secretion-capture role for apolipoprotein E in remnant lipoprotein metabolism involving cell surface heparan sulfate proteoglycans. J. Biol. Chem. 269:2764-2772.

28. Ji, Z.-S., Sanan, D.A., and Mahley, R.W. 1995 Intravenous heparinase inhibits remnant lipoprotein clearance from the plasma and uptake by the liver: in vivo role of heparan sulfate proteoglycans. J. Lipid Res. 36:583-592.

29. Fuki, I.V., et al. 1997. The syndecan family of proteoglycans. Novel receptors mediating internalization of atherogenic lipoproteins in vitro. J. Clin. Invest. 100:1611-1622.

30. Zeng, B.-J., Mortimer, B.-C., Martins, I.J., Seydel, U., and Redgrave, T.G. 1998. Chylomicron remnant uptake is regulated by the expression and function of heparan sulfate proteoglycan in hepatocytes. J. Lipid Res. 39:845-860.

31. Wilsie, L.C., and Orlando, R.A. 2003. The low density lipoprotein receptor-related protein complexes with cell surface heparan sulfate proteoglycans to regulate proteoglycan-mediated lipoprotein catabolism. J. Biol. Chem. 278:15758-15764.

32. MacArthur, J.M., et al. 2007. Liver heparan sulfate proteoglycans mediate clearance of triglyceride-rich lipoproteins independently of LDL receptor family members. J. Clin. Invest. 117:153-164. doi:10.1172/ JCI29154.

33. Bernfield, M., et al. 1999. Functions of cell surface heparan sulfate proteoglycans. Annu. Rev. Biochem. 68:729-777.

34. Esko, J.D., and Selleck, S.B. 2002. Order out of chaos: assembly of ligand binding sites in heparan sulfate. Annu. Rev. Biochem. 71:435-471.

35. Jones, C., et al. 2007. Disruption of LDL but not VLDL clearance in autosomal recessive hypercholesterolemia. J. Clin. Invest. 117:165-174. doi:10.1172/JCI29415.

36. Garcia, C.K., et al. 2001. Autosomal recessive hypercholesterolemia caused by mutations in a putative LDL receptor adaptor protein. Science. 292:1394-1398.

37. Jones, C., et al. 2003. Normal sorting but defective endocytosis of the low density lipoprotein receptor in mice with autosomal recessive hypercholesterolemia. J. Biol. Chem. 278:29024-29030.

38. He, G., et al. 2002. ARH is a modular adaptor protein that interacts with the LDL receptor, clathrin, and AP-2. J. Biol. Chem. 277:44044-44049. 